\title{
Control of 12-Cylinder Camless Engine with Neural Networks
}

\author{
"Moh'd Sami” Ashhab \\ Department of Mechanical and Industrial Engineering, American University of Ras Al Khaimah, UAE \\ Department of Mechanical Engineering, Hashemite University, Zarqa, Jordan
}

\begin{abstract}
The 12-cyliner camless engine breathing process is modeled with artificial neural networks (ANN's). The inputs to the net are the intake valve lift (IVL) and intake valve closing timing (IVC) whereas the output of the net is the cylinder air charge (CAC). The ANN is trained with data collected from an engine simulation model which is based on thermodynamics principles and calibrated against real engine data. A method for adapting single-output feed-forward neural networks is proposed and applied to the camless engine ANN model. As a consequence the overall 12-cyliner camless engine feedback controller is upgraded and the necessary changes are implemented in order to contain the adaptive neural network with the objective of tracking the cylinder air charge (driver's torque demand) while minimizing the pumping losses (increasing engine efficiency). All the needed measurements are extracted only from the two conventional and inexpensive sensors, namely, the mass air flow through the throttle body (MAF) and the intake manifold absolute pressure (MAP) sensors. The feedback controller's capability is demonstrated through computer simulation.
\end{abstract}

\section{Introduction}

One of the major challenges in the camless IC engine design is controlling the cylinder air charge (CAC) rapidly and accurately based on conventional measurements. A feedback control system that is responsible for this task has been developed in [1]. It consists of a feedforward controller, a CAC estimator and an on-line parameter estimator which is used to adapt the feedforward controller parameters using the CAC estimate. The performance of the controller requires accurate estimation of the CAC. It is very difficult to measure the cylinder air charge directly since there are no sensors that can be placed inside or close to the cylinders due to the high pressure and temperature of the cylinder. Thus, the CAC must be estimated based on conventional engine measurements.

It has been shown in [1] that the individual cylinder air charge measurements can be extracted from two conventional and inexpensive sensors, namely, the mass air flow through the throttle body (MAF) and the intake manifold absolute pressure (MAP) sensors. The estimated cylinder air charge is used therein to update the feedforward controller parameters via the on-line parameter estimator in order to account for modeling errors and engine parts degradation. The adaptation technique is based on shifting and scaling the inputs to the engine. These inputs are the intake valve lift (IVL) and intake valve closing timing (IVC) and they are set by electrohydraulic actuators. One disadvantage of the feedforward controller is that its operation is based on graphical techniques. In addition, the camless engine controller design becomes more challenging for a larger number of cylinders such as a 12-cylinder engine.

In this research, the 12-cyliner camless engine breathing process is modeled with artificial neural networks (ANN's). The inputs to the net are the IVL and IVC whereas the output of the net is the CAC. The ANN is trained with engine input output data at a constant speed of $1500 \mathrm{rpm}$. The ANN model forecasts the CAC in the future at this speed. It has been shown in [2] that the feedforward controller developed in [1] can be modeled by inverting the camless engine ANN model. The ANN based feedforward controller is more concise and easier to adapt. A method for adapting single-output feed-forward neural networks is proposed in this research and applied to the camless engine ANN model. As a consequence the overall 12-cyliner camless engine feedback controller is upgraded and the necessary changes are implemented in order to contain the adaptive neural network with the objective of tracking the cylinder air charge (driver's torque demand) while minimizing the pumping losses. The feedback controller's capability is demonstrated through computer simulation.

The camless engine model was initiated in [3] as a result of collaboration between academia and industry. Furthermore, the basic control system of such an engine was designed in [1]. Improvements of the initial control system design that enhanced the performance of the system through computer simulation were attempted later. In [2], graphical methods for the feedforward controller were replaced by inverting a neural network model for the engine. Adapting the neural network achieved good long term performance of the camless engine that 
accommodates the changing environment and aging [4]. Analysis of the updated camless engine controller can be found in $[5,6]$. The 4-cylinder camless engine with the fully updated controller was presented in [7]. Similarly, the 8-cylinder engine with its updated controller was studied in [8]. Modeling and control of the 12-cylinder engine will be conducted in this research which represents the basis for this engine operation.

The camless engine performance depends on the design of electrohydraulic actuators for regulating the valve motion. Researchers have attempted different designs for the electrohydraulic actuators to achieve the best engine performance. Application of a robust motion control technique to an electrohydraulic camless engine valve actuator was presented in [9]. The research of [10] deals with a signal-based robust adaptive approximation technique for a proportional derivative (PD) regulator which is applied to an electromagnetic valve actuator control for camless internal combustion engines. Exhaust valve motion was analyzed towards optimum performance in [11].

Artificial neural networks (ANN's) have received a lot of attention in recent years due to their attractive capabilities in forecasting, modeling of complex nonlinear systems and control. Applications of neural networks include many engineering fields. For example, ANN's have been used for forecasting load [12] and glass/epoxy failure strength [13].

\section{Intake process analysis}

The camless engine is an internal combustion engine that operates without a camshaft. The intake valves are controlled by electrohydraulic actuators that activate the valves motion. In the case of the camless engine the valve motion is not tied to the engine rotation but it is set by an engine controller instead. Consequently the choice of the intake valve motion is very flexible which allows efficient engine performance characterized by satisfying the desired cylinder air charge (driver torque demand) and minimizing pumping losses (fuel economy) at all speeds. In order to design the appropriate controller that achieves these objectives, the engine breathing (intake) process needs to be analyzed and studied.

The thermodynamic equations that describe the intake process of internal combustion engines including the 12cyliner camless engine are based on the principles of the conservation of mass and the ideal gas law [3]. More specifically, the following relations hold for the engine cylinders

$$
\begin{aligned}
& \frac{d p_{m}}{d t}=\frac{R T}{V_{m}}\left(\dot{m}_{\varphi}-\sum_{i=1}^{n} \dot{m}_{i}\right) \\
& \frac{d p_{i}}{d t}=\frac{1}{V_{i}}\left(R T \dot{m}_{i}-\dot{V}_{i} p_{i}\right)
\end{aligned}
$$

where $n$ is the number of cylinders, subscript $m$ refers to manifold, subscript $i$ refers to cylinder $i(i=1,2 \ldots n), p$ is the pressure, $t$ is the time, $V$ is the volume, $T$ is the

temperature, $\mathrm{R}$ is the gas constant, and $\dot{m}$ is the mass flow rate. The subscript $\phi$ refers to the manifold throttle. In the camless engine the manifold throttle is widely open all the time and not connected to the gas pedal since the air flow into the cylinders is controlled by the individual cylinder intake valves. On the other hand, the throttle opening changes in the conventional engine according to the driver demand due to the fact that this is the only means of controlling the air flow into the cylinders. The intake valve motion is fixed with the engine rotation in the conventional engine.

A simulation model in Matlab software was built for the engine based on Equations (1) and (2) and implementing the implicit relations of the terms within the equations [3]. The various implicit relations include parameters that needs to be selected by comparison with real experimental engine data available from the dynamometer test at an automotive company. From the engine equations the pressure versus crank angle (or time) are plotted and compared with real engine data under the same conditions. During this process the parameters of the engine mathematical model are set such that there is a good match between the simulation results and real experimental engine data. An example of such a comparison is given in Figure 1.

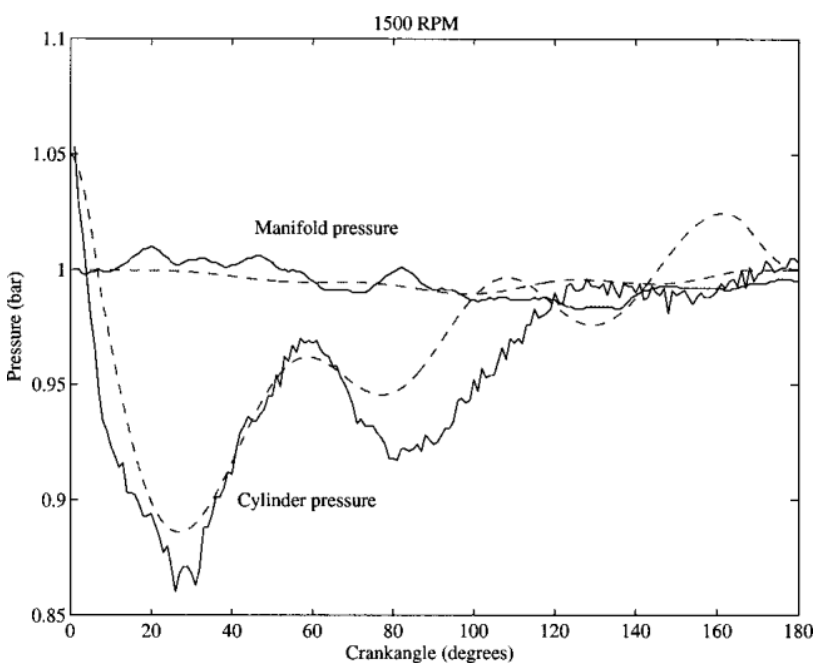

Figure 1. Simulation model versus experimental data. The continuous line represents the experimental data whereas the dashed line represents the model [1].

\section{Neural net model}

The breathing process of the 12-cylinder camless engine was modeled in [14] with artificial neural networks (ANN's) and brief results are given here. The neural net structure to be used for modeling the camless engine breathing process is shown in Figure 2. The inputs are the intake valve lift (IVL) and intake valve closing timing (IVC) whereas the outputs are the important variables for control design, namely, the cylinder air charge (CAC) and pumping loss (PL). CAC needs to be tracked based 
on the driver's torque demand while minimizing the pumping loss. IVL and IVC feed forward through a hidden layer to the CAC and PL.

An artificial neural net mathematical model that represents the structure shown in Figure 2 is written as

$$
Y=f(U)=W_{o} * \tanh \left(W_{i} * U+B_{i}\right)+B_{o}
$$

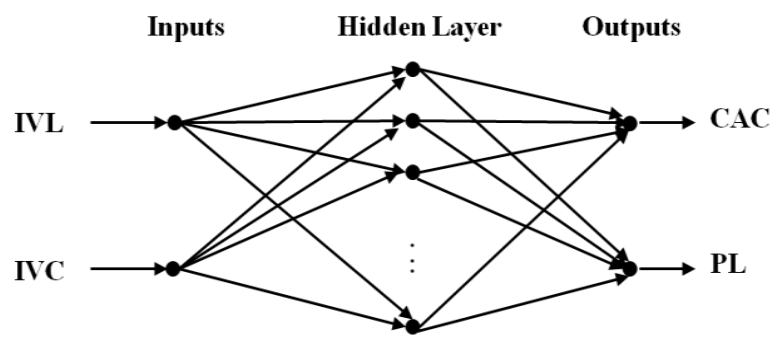

Figure 2. Camless engine neural net structure.

where, $Y$ is a column vector which contains the 2 outputs of the process, $U$ is a column vector that contains the 2 inputs of the process, $W_{o}$ is a matrix of size $2 \mathrm{x} n$ that contains the weights of the neural net model from the hidden layer to the outputs with $n$ being the number of neurons in the hidden layer, $W_{i}$ is a matrix of size $n \times 2$ that contains the weights of the neural net model from the inputs to the hidden layer, $B_{i}$ (not shown in Figure 2) is a column vector of size $n$ that contains the biases from the inputs to the hidden layer and $B_{o}$ (not shown in Figure 2) is a column vector of size 2 which contains the biases from the hidden layer to the outputs.

The cylinder air charge from the ANN is the first element of $Y$ and thus Equation (3) can be used to write

$$
\mathrm{CAC}(t+1)=\beta(U(t)) \alpha)
$$

where,

$$
\begin{gathered}
\beta(U(t))=\left[\begin{array}{c}
\tanh \left(W_{i}^{*} U(t)+B_{i}\right) \\
1
\end{array}\right]^{\mathrm{T}} \\
\alpha=\left[\begin{array}{c}
W_{o 1}^{\mathrm{T}} \\
B_{o 1}
\end{array}\right]
\end{gathered}
$$

with $W_{o 1}$ being the first row of $W_{o}$ and $B_{o 1}$ the first element of $B_{o}$. It is clear that the estimated (neural network) future value of CAC is linear in the parameter $\alpha$ that is a column composed of the output weight vector and bias that correspond to CAC.

\section{Feedback adaptive controller}

One of the major challenges in the camless IC engine design is controlling the cylinder air charge (CAC) rapidly and accurately based on conventional measurements while minimizing the pumping loss (PL). The feedback control system that is responsible for this task is shown in Figure 3. It consists of a feedforward controller, a CAC estimator and an on-line parameter estimator which is used to adapt the feedforward controller parameters using the CAC estimate. It has been shown in [1] that the individual cylinder air charge measurements can be extracted from two conventional and inexpensive sensors, namely, the mass air flow through the throttle body (MAF) and the intake manifold absolute pressure (MAP) sensors. The estimated cylinder air charge is used therein to update the feedforward controller parameters via the on-line parameter estimator in order to account for modeling errors and engine parts degradation. The adaptation technique is based on shifting and scaling the inputs to the engine. These inputs are the intake valve lift (IVL) and intake valve closing timing (IVC) and they are set by electrohydraulic actuators. Two disadvantages of this feedforward controller are: 1) its operation is based on graphical techniques and 2) large amount of engine input-output data at various speeds needs to be stored in controller's memory.

The ANN is used to predict the cylinder air charge in the future. Due to possible gradual degradation of the camless engine, the short-term predictions will be more accurate than the long-term ones. The neural net of the camless engine is adapted in [4] using the Kaczmarz's algorithm in order to better accommodate the changing environment and improve the net's prediction accuracy over the long run. This adaptation scheme leads to less computational complexity and quick convergence. The real-time parameter estimation Kaczmarz's algorithm when applied to the ANN model described by Equation (4) gives the following recursive adaptation scheme for $\alpha$

$$
\alpha(t+1)=\alpha(t)+\frac{\beta(U(t-1))^{\mathrm{T}}}{\beta(U(t-1)) \beta(U(t-1))^{\mathrm{T}}} e
$$

where the error, $e$, is the difference between the real and ANN estimated cylinder air charge at the time instant $t$.

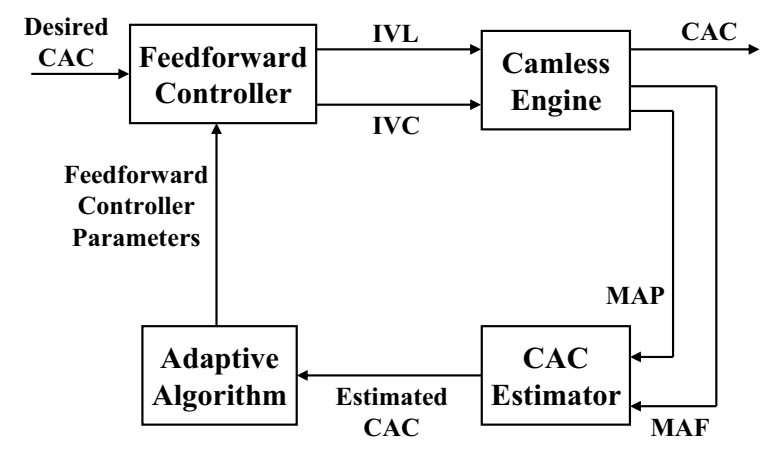

Figure 3. Camless engine adaptive feedback controller.

Note that the on-line adaptive algorithm described in Equation (7) assumes that the output cylinder air charge measurement at the current time $t$ is available which is 
true in the camless engine. The estimated output of the ANN model is compared with the measured cylinder air charge of the engine. If there is a difference between the two values the parameter estimation Kaczmarz's algorithm updates $\alpha$ (according to Equation (7)) to be used in the subsequent predictions. The parameter estimator or adaptive algorithm compensates for engine parts degradation and modeling errors, and provides more accurate long-term predictions.

\section{Simulation Results}

The capability of the developed ANN based adaptive 12cylinder camless IC engine controller is tested with the aid of computer simulation. Similar tests were performed for the 8-cylinder engine in [8]. However, the 12-cylinder engine case is more complicated due to the fact that there is a higher probability of overlapping cylinders during engine operation. We test three different cases. In the first case the controller tracks changing desired cylinder air charge. In the second case the controller is tested as the speed changes from 1500 RPM to 1700 RPM. Finally, in the third case deposits are assumed to be present on the intake valves.

In the first case of 1500 RPM engine speed, the controller achieves cylinder air charge tracking as it changes from $0.3 \mathrm{~g}$ to $0.4 \mathrm{~g}$ at 0.3 seconds, in addition to balancing by eliminating modeling errors as shown in Figure 4. Note that the 12 cylinders are plotted with different styles. The desired cylinder air charge is the solid line. The operation of the controller in this case depends almost completely on the feedforward controller that is the inverse of the camless engine ANN model. The feedforward controller selects the intake valve lift (IVL) and closing timing (IVC) that will assure that the cylinder air charge is equal to its desired value for the nominal situation. We have here a nominal engine since the speed is the same speed for which the ANN has been trained and there is no parts degradation or noise introduced in the system. A moderate adaptation effort is required to compensate for modeling errors. We conclude that the ANN based feedforward controller achieves its objective of satisfying the desired cylinder air charge.

The camless engine ANN model was trained using data at a speed of 1500 RPM. The feedforward controller performance will not satisfy the cylinder air charge demand at other speeds. The adaptive controller compensates for speed variations as shown in Figure 5. The speed changes from $1500 \mathrm{RPM}$ to $1700 \mathrm{RPM}$ at $t=$ 0.24 seconds and the feedforward controller selects initially IVL and IVC from the nominal 1500 RPM data. These values fail to satisfy the desired cylinder air charge of $0.3 \mathrm{~g}$ and provide instead a lower cylinder air charge. The predicted cylinder air charge of the ANN model (approximately equals $0.3 \mathrm{~g}$ ) is compared with the measured cylinder air charge of the engine which is estimated via the cylinder air charge estimator. The parameter estimation Kaczmarz's algorithm uses the error between the two values to update the ANN parameters for the current speed of 1700 RPM to be used in the subsequent predictions and feedforward controller. The adaptive controller achieves the desired cylinder air charge in one engine cycle.
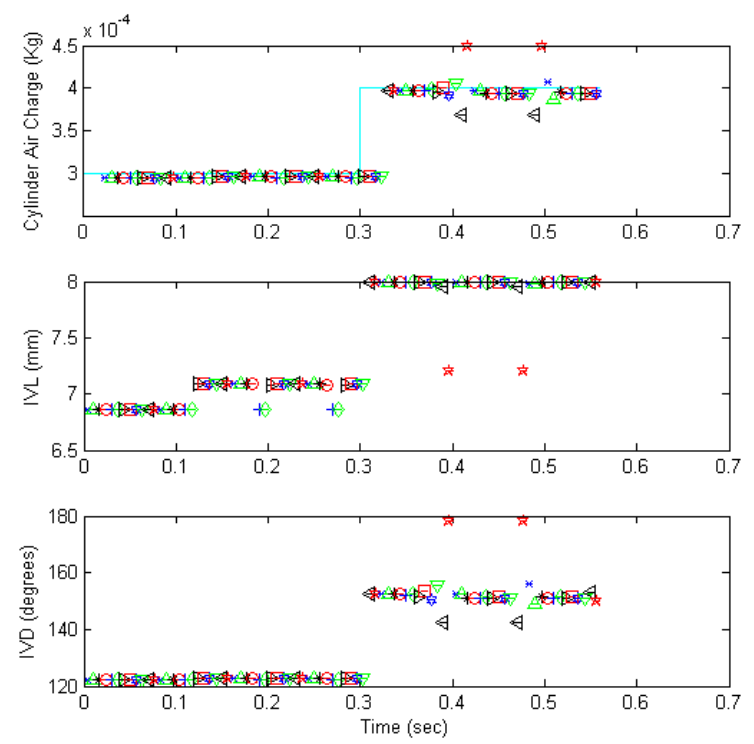

Figure 4. Cylinder air charge tracking at a fixed speed of 1500 RPM.

The designed adaptive feedback controller can compensate for engine parts degradation and thus keeps consistent and good engine performance over the long time horizon. We assume that intake valves allow less air into the cylinders due to deposits that accumulate with time. It is assumed that the effective area of the valve (through which air passes from the manifold to the cylinder) is reduced by $5 \%$ for all of the 12 cylinders due to the deposits. The simulation results of this case are shown in Figure 6. Note that the adaptive feedback controller overcomes the valve degradation and keeps the engine performance at the same high level. The feedforward controller initially does not satisfy the cylinder air charge. The deposits cause a CAC reduction. The adaptive algorithm uses the error in CAC to compensate for the degradation and the controller achieves CAC tracking in one engine cycle.
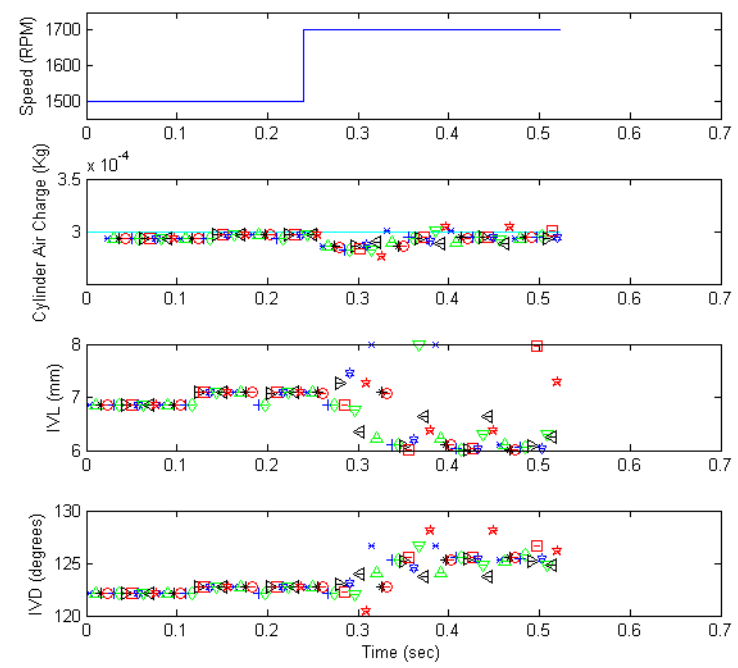

Figure 5. Cylinder air charge tracking with speed variation. 


\section{Conclusions}

An adaptive feedback artificial neural net (ANN) based camless 12-cylinder engine controller with the objective of achieving the desired cylinder air charge (CAC) or driver's torque demand and minimizing the pumping loss (PL) was developed. The controller consists of a feedforward controller, cylinder air charge estimator, and on-line ANN parameter estimator. The feedforward controller is the inverse of the ANN camless 12-cylinder engine model which was developed at a speed of 1500 RPM. The feedforward controller provides the intake valve lift (IVL) and intake valve closing timing (IVC) that satisfy the desired CAC and minimize PL. The parameters of the feedforward controller are updated recursively using the error between the cylinder air charge measurement from the cylinder air charge estimator and its predicted value from the ANN. The adaptation scheme improves the ANN prediction accuracy when the engine parts degrade, speed changes and in the presence of modeling errors. The camless engine controller achieves its objective of CAC tracking and minimizing PL.
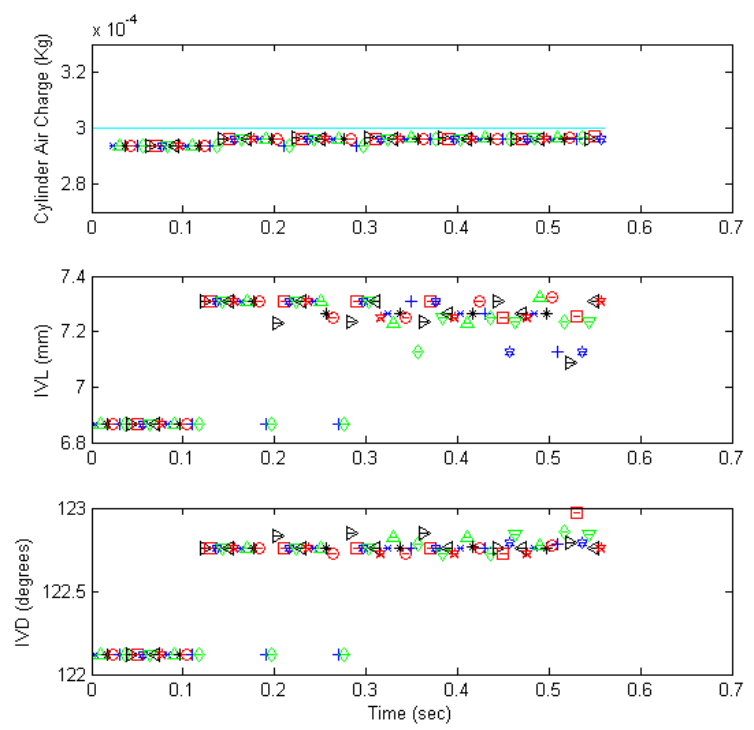

Figure 6. Cylinder air charge tracking with deposits on valves.

\section{References}

1. M. Ashhab, A. Stefanopoulou, J. Cook, M. Levin, Journal of Dynamic Systems, Measurement, and Control 122, 131 (2000)

2. M. S. Ashhab, Fourth IASTED International Conference on Modeling, Simulation and Optimization, Kauai, USA (2004)

3. M. Ashhab, A. Stefanopoulou, J. Cook, M. Levin, Journal of Dynamic Systems, Measurement, and Control 122, 122 (2000)

4. M. S. Ashhab, Fifth IASTED International Conference on Modeling, Simulation and Optimization, Oranjestad, Aruba (2005)
5. M. S. Ashhab, International Conference on Dynamics, Instrumentation and Control, Queretaro, Mexico (2006)

6. M .S. Ashhab, Energy Conversion and Management 49, 365 (2008)

7. M. S. Ashhab, IASTED International Conference on Applied Simulation and Modeling, Palma de Mallorca, Spain (2007)

8. M. S. Ashhab, Second International Conference on Kansei Engineering \& Affective Systems, Nagaoka, Japan (2008)

9. Y. Yoon, Z. Sun, IEEE Transactions on Industrial Electronics 63, 5724 (2016)

10. P. Mercorelli, Asian Journal of Control 18, 1299 (2016)

11. X. Y. Fan, L. Liu, S. Q. Chang, J. T. Xu, J. G. Dai, International Journal of Automotive Technology 17, 361 (2016)

12. J. Massana, C. Pous, L. Burgas, J. Melendez, J. Colomer, Energy and Buildings 92, 322 (2015)

13. C. Suresh Kumar, V. Arumugam, R. Sengottuvelusamy, S. Srinivasan, H. N. Dhakal, Applied Acoustics 115, 32 (2017)

14. M. S. Ashhab, International Journal of Automation, Mechatronics \& Robotics 3, 99 (2016) 